\title{
Comparison on Trapezoidal and Simpson’s Rule for Unequal Data Space
}

\author{
Md. Nayan Dhali ${ }^{\mathrm{a}}$, Mohammad Farhad Bulbul ${ }^{\mathrm{b}^{*}}$ and Umme Sadiya ${ }^{\mathrm{c}}$ \\ ${ }^{a, b, c}$ Department of Mathematics, Jashore University of Science and Technology, Jashore-7408, Bangladesh
}

Received: 07 April 2019; Accepted: 14 June 2019; Published: 08 November 2019

\begin{abstract}
Numerical integration compromises a broad family of algorithm for calculating the numerical value of a definite integral. Since some of the integration cannot be solved analytically, numerical integration is the most popular way to obtain the solution. Many different methods are applied and used in an attempt to solve numerical integration for unequal data space. Trapezoidal and Simpson's rule are widely used to solve numerical integration problems. Our paper mainly concentrates on identifying the method which provides more accurate result. In order to accomplish the exactness we use some numerical examples and find their solutions. Then we compare them with the analytical result and calculate their corresponding error. The minimum error represents the best method. The numerical solutions are in good agreement with the exact result and get a higher accuracy in the solutions.
\end{abstract}

Index Terms: Numerical Integration; Trapezoidal Rule; Simpson’s Rule.

(C) 2019 Published by MECS Publisher. Selection and/or peer review under responsibility of the Research Association of Modern Education and Computer Science

\section{Introduction}

Numerical integration is the approximate computation of an integral using numerical technique. The concept of numerical integration is very important in Mathematics. Numerical integration is a primary tool used by engineers and scientists to obtain an approximate result for definite integrals that cannot be solved analytically. Initial value problem and boundary value problems involving either ordinary or partial differential equations, may be solved by numerical integrations. Numerical solutions do not usually allow the determinations of general physics laws and do not often indicate the dependence of the desired variables on the various parameter of the problem. If is a smooth function integrand over a definite interval, there are many numerical methods for

* Corresponding author.

E-mail address: 
approximating the integral for unequal data points. The question arises which numerical method gives more accurate result. Here we apply different numerical integration formula for unequal data space and determine the desire numerical method.

Romesh Kumar Muthumalai tried to determine the error of numerical integration and differentiation and he also derived some formula for numerical differentiation through divided difference and these new formulas are quite useful to approximate derivatives when additional information about derivatives at some point is given. Md. Mamun-Ur -Rashid khan, M.R. Hossain and Selina Parvin developed a new approach of numerical integration schemes for unequal data space. Rajinder thukral accelerated the Simpson's method for solving nonlinear equations. In this purpose he represented an improvement of the classical Simpson's third order method for finding zeros of nonlinear equations and introduced a new formula for approximating second order derivative. J Jayakumar generalized Simpson's, newton method for solving nonlinear equation with cubic convergence. For that, a new class of Newton's method for solving a single nonlinear equation is proposed $[1,2,3,4]$. Hamidah Eskandari developed Simpson's method for solution of nonlinear equation. He proposed the integration method for finding the root of nonlinear equation [12].

The rest of the paper is organized as follows. In Section two, we present general quadrature formula for unequal space. In section three, we discuss Trapezoidal and Simpson's rule for unequal space. In section four, we discuss some numerical examples. Finally, we draw a conclusion.

\section{General Quadrature Formula for Unequal Space}

Consider a definite interval

$$
I=\int_{x_{0}}^{x_{n}} y d x
$$

Where the interval $\left[x_{0}, x_{n}\right]$ be divided $n$ unequally subinterval. The distances of each of the interval are not equal.

Let $\left(x_{0}, y_{0}\right),\left(x_{1}, y_{1}\right),\left(x_{2}, y_{2}\right), \ldots \ldots \ldots,\left(x_{n}, y_{n}\right)$ be the given $(n+1)$ points then by Newton`s divided difference formula, we have

$$
y=y_{0}+\left(x-x_{0}\right)\left[x_{0}, x_{1}\right]+\left(x-x_{0}\right)\left[x-x_{1}\right]\left[x_{0}, x_{1}, x_{2}\right]+\ldots \ldots
$$

So, From (1)

$$
\begin{aligned}
& \int_{x_{0}}^{x_{n}} y d x=\int_{x_{0}}^{x_{n}}\left(y_{0}+\left(x-x_{0}\right)\left[x_{0}, x_{1}\right]+\left(x-x_{0}\right)\left(x-x_{1}\right)\left[x_{0}, x_{1}, x_{2}\right]+\ldots\right) d x \\
& =\left[y_{0} x+\frac{\left(x-x_{0}\right)^{2}}{2}\left[x_{0}, x_{1}\right]\right]_{x_{0}}^{x_{n}}+\frac{1}{2}\left[x_{0}, x_{1}, x_{2}\right] \int_{x_{0}}^{x_{n}}\left(x-x_{0}\right) d\left(x-x_{1}\right)^{2} d x+\ldots . . \\
& \left.=y_{0}\left(x_{n}-x_{0}\right)+\frac{\left(x_{n}-x_{0}\right)^{2}}{2}\left[x_{0}, x_{1}\right]+\frac{1}{2}\left[x_{0}, x_{1}, x_{2}\right]\left[\left(x-x_{0}\right)\left(x-x_{1}\right)^{2}\right]_{x_{0}}^{x_{n}}-\int_{x_{0}}^{x_{n}}\left(x-x_{1}\right)^{2} d x\right]+\ldots
\end{aligned}
$$

Thus, the equation of general quadrature formula for unequal data space is 


$$
\begin{aligned}
& \int_{x_{0}}^{x_{n}} y d x=y_{0}\left(x_{n}-x_{0}\right)+\frac{\left(x_{n}-x_{0}\right)^{2}}{2}\left[x_{0}, x_{1}\right]+\frac{1}{2}\left[x_{0}, x_{1}, x_{2}\right]\left[\left(x_{n}-x_{0}\right)\left(x_{n}-x_{1}\right)^{2}-\frac{1}{3}\left(x_{n}-x_{1}\right)^{3}\right. \\
& \left.+\frac{1}{3}\left(x_{0}-x_{1}\right)^{3}\right]+\ldots . .
\end{aligned}
$$

\section{Numerical Integration Methods}

To By Eqn. (2), we can obtain different integration formulae for $n=1,2,3$. etc. We derive here a numerical formula for the Trapezoidal rule, Simpson's $\frac{1}{3}$ rule and Simpson's $\frac{3}{8}$ rule.

\subsection{Trapezoidal Rule}

Putting $n=1$ in Eqn. ${ }^{(2)}$ then we got for the interval $\left[x_{0}, x_{1}\right]$

$\int_{x_{0}}^{x_{1}} y d x=y_{0}\left(x_{1}-x_{0}\right)+\frac{\left(x_{1}-x_{0}\right)^{2}}{2}\left[x_{0}, x_{1}\right]=\frac{\left(x_{1}-x_{0}\right)}{2}\left[y_{0}+y_{1}\right]$

Similarly, for intervals $\left[x_{1}, x_{2}\right],\left[x_{2}, x_{3}\right] \ldots\left[x_{n-1}, x_{n}\right]$ we compute the integrals and adding all these terms. Then we got the following general formula of trapezoidal rule for unequal space.

$$
\int_{x_{0}}^{x_{n}} y d x=\frac{1}{2}\left[\left(x_{1}-x_{0}\right) y_{0}+\left(x_{2}-x_{0}\right) y_{1}+\left(x_{3}-x_{1}\right) y_{2}+\ldots+\left(x_{n}-x_{n-2}\right) y_{n-1}+\left(x_{n}-x_{n-1}\right) y_{n}\right]
$$

\subsection{Simpson`s 1/3 Rule}

Putting $n=2$ in Eqn. (2) then we got for the interval $\left[x_{0}, x_{2}\right]$

$\int_{x_{0}}^{x_{2}} y d x=y_{0}\left(x_{2}-x_{0}\right)+\frac{\left(x_{2}-x_{0}\right)^{2}}{2}\left[x_{0}, x_{1}\right]+\frac{1}{2}\left[x_{0}, x_{1}, x_{2}\right]\left(\left(x_{2}-x_{0}\right)\left(x_{2}-x_{1}\right)^{2}-\frac{1}{3}\left(x_{2}-x_{1}\right)^{3}+\frac{1}{3}\left(x_{0}-x_{1}\right)^{3}\right)$

Similarly, for intervals $\left[x_{2}, x_{4}\right] \ldots\left[x_{n-2}, x_{n}\right]$ we compute the integrals and adding all these terms. Then we got the following general formula of Simpson`s $\frac{1}{3}$ - rule for unequal space.

$$
\begin{aligned}
& \int_{x_{0}}^{x_{n}} y d x=\sum_{i=2}^{n} y_{i-2}\left(x_{i}-x_{i-2}\right)+\frac{\left(x_{i}-x_{i-2}\right)^{2}}{2}\left[x_{i-2}, x_{i-1}\right]+\frac{1}{2}\left[x_{i-2}, x_{i-1}, x_{i}\right]\left(\left(x_{i}-x_{i-2}\right)\left(x_{i}-x_{i-1}\right)^{2}-\frac{1}{3}\left(x_{i}-x_{i-1}\right)^{3}\right. \\
& \left.+\frac{1}{3}\left(x_{i-2}-x_{i-1}\right)^{3}\right)
\end{aligned}
$$




\subsection{Simpsons 3/8 Rule}

Putting $n=3$ in Eqn. (2) then we got for the interval $\left[x_{0}, x_{3}\right]$

$$
\begin{aligned}
& \int_{x_{0}}^{x_{3}} y d x=y_{0}\left(x_{3}-x_{0}\right)+\frac{\left(x_{3}-x_{0}\right)^{2}}{2}\left[x_{0}, x_{1}\right]+\frac{1}{2}\left[x_{0}, x_{1}, x_{2}\right]\left(\left(x_{3}-x_{0}\right)\left(x_{3}-x_{1}\right)^{2}-\frac{1}{3}\left(x_{3}-x_{1}\right)^{3}+\frac{1}{3}\left(x_{0}-x_{1}\right)^{3}\right) \\
& +\frac{1}{2}\left[x_{0}, x_{1}, x_{2}, x_{3}\right]\left(\left(x_{3}-x_{0}\right)\left(x_{3}-x_{1}\right)\left(x_{3}-x_{2}\right)^{2}-\frac{1}{3}\left(x_{3}-x_{1}\right)\left(x_{3}-x_{2}\right)^{3}+\frac{1}{3}\left(x_{0}-x_{1}\right)\left(x_{0}-x_{2}\right)^{3}\right)+\frac{1}{12}\left(x_{3}-x_{2}\right)^{4} \\
& -\frac{1}{12}\left(x_{0}-x_{2}\right)^{4}
\end{aligned}
$$

Similarly for intervals $\left[x_{3}, x_{6}\right] \ldots\left[x_{n-3}, x_{n}\right]$ we compute the integrals and adding all these terms. Then we got the following general formula of Simpson`s $\frac{1}{3}$ - rule for unequal space.

$$
\begin{aligned}
& \int_{x_{0}}^{x_{n}} y d x=\sum_{i=3}^{n} y_{i-3}\left(x_{i}-x_{i-3}\right)+\frac{\left(x_{i}-x_{i-3}\right)^{2}}{2}\left[x_{i-3}, x_{i-2}\right]+\frac{1}{2}\left[x_{i-3}, x_{i-2}, x_{i-1}\right]\left(\left(x_{i}-x_{i-3}\right)\left(x_{i}-x_{i-2}\right)^{2}-\frac{1}{3}\left(x_{i}-x_{i-2}\right)^{3}\right. \\
& \left.+\frac{1}{3}\left(x_{i-3}-x_{i-2}\right)^{3}\right)+\frac{1}{2}\left[x_{i-3}, x_{i-2}, x_{i-1}, x_{i}\right]\left(\left(x_{i}-x_{i-3}\right)\left(x_{i}-x_{i-2}\right)\left(x_{i}-x_{i-1}\right)^{2}-\frac{1}{3}\left(x_{i}-x_{i-2}\right)\left(x_{i}-x_{i-1}\right)^{3}\right. \\
& \left.+\frac{1}{3}\left(x_{i-3}-x_{i-2}\right)\left(x_{i-3}-x_{i-1}\right)^{3}\right)+\frac{1}{12}\left(x_{i}-x_{i-1}\right)^{4}-\frac{1}{12}\left(x_{i-3}-x_{i-1}\right)^{4}
\end{aligned}
$$

\section{Results \& Discussion}

In this section, we discuss some numerical examples and using numerical integration formula for unequal data space and we try to find their solutions. We then compare numerical result with the exact solution to determine corresponding errors and check out which methods provide better result.

\subsection{Investigation by First Example}

Consider the integration

$$
f(x)=\int_{0}^{4} \frac{x^{3} d x}{\sqrt{x^{2}+9}}
$$

We consider $60 \mathrm{sub}$ intervals over the interval 0 to 4 .

$$
\text { Table } 1 \text { represents the values of } f(x)=\int_{0}^{4} \frac{x^{3} d x}{\sqrt{x^{2}+9}} \text { for different values of } x
$$

Now, we determine the solution of the first problem by using Trapezoidal, Simpson's $\frac{1}{3}$ and Simpson's $\frac{3}{8}$ rule for unequal data space and compare the result with exact result. The above result is given bellow. 
Table 1. Calculating the values of $f(x)$ for random values of $x$

\begin{tabular}{|c|c|c|c|c|c|}
\hline Value of $X$ & Value of $f(x)$ & Value of $X$ & Value of $f(x)$ & Value of $X$ & Value of $f(x)$ \\
\hline 0 & 0 & 0.25 & 0.005190342 & 0.8 & 0.164904096 \\
\hline 0.1 & 0.000333148 & 0.26 & 0.005836787 & 0.9 & 0.232751787 \\
\hline 0.12 & 0.00057554 & 0.27 & 0.006534588 & 1 & 0.316227766 \\
\hline 0.13 & 0.000731647 & 0.29 & 0.008091947 & 1.2 & 0.534802574 \\
\hline 0.15 & 0.001123596 & 0.3 & 0.008955335 & 1.4 & 0.828855512 \\
\hline 0.16 & 0.001363396 & 0.32 & 0.010861054 & 1.6 & 1.204705882 \\
\hline 0.17 & 0.001635044 & 0.4 & 0.021146195 & 1.8 & 1.666966248 \\
\hline 0.18 & 0.00194051 & 0.43 & 0.02623422 & 2 & 2.218800785 \\
\hline 0.2 & 0.00266076 & 0.5 & 0.041099747 & 2.1 & 2.528968939 \\
\hline 0.23 & 0.0040438 & 0.6 & 0.070601809 & 2.4 & 3.598243474 \\
\hline 0.24 & 0.004593325 & 0.7 & 0.111342512 & 2.6 & 4.42733044 \\
\hline 2.7 & 4.876752894 & 3.2 & 7.47046097 & 3.37 & 8.482695567 \\
\hline 2.8 & 5.349375083 & 3.21 & 7.528185604 & 3.4 & 8.668125608 \\
\hline 2.9 & 5.845135834 & 3.24 & 7.702726052 & 3.5 & 9.300893379 \\
\hline 3 & 6.363961031 & 3.25 & 7.761361384 & 3.6 & 9.956147784 \\
\hline 3.1 & 6.905766582 & 3.27 & 7.879314222 & 3.7 & 10.6337862 \\
\hline 3.11 & 6.961207586 & 3.3 & 8.057947399 & 3.78 & 11.19194516 \\
\hline 3.12 & 7.016877387 & 3.31 & 8.117945766 & 3.9 & 12.05581087 \\
\hline 3.14 & 7.128903001 & 3.33 & 8.23862285 & 4 & 12.8 \\
\hline 3.15 & 7.185258621 & 3.34 & 8.299301364 & & \\
\hline 3.17 & 7.298655004 & 3.36 & 8.421337825 & & \\
\hline
\end{tabular}

Table 2. Comparison between exact and numerical result

\begin{tabular}{|c|c|c|c|}
\hline Method & Result & Exact value & Error \\
\hline Trapezoidal & 14.6833 & & $0.11318 \%$ \\
Simpsons $1 / 3$ & 14.6666 & 14.6667 & $0.00068 \%$ \\
Simpsons 3/8 & 14.6911 & & $0.16636 \%$ \\
\hline
\end{tabular}

Table 2 shows the comparison of Trapezoidal rule Simpson's $\frac{1}{3}$ and Simpson's $\frac{3}{8}$

Table 2 shows the comparison of Trapezoidal rule, Simpson's $\overline{3}$ and Simpson's $\overline{8}$ rule. Now, we describe the above comparison by the following diagram. 


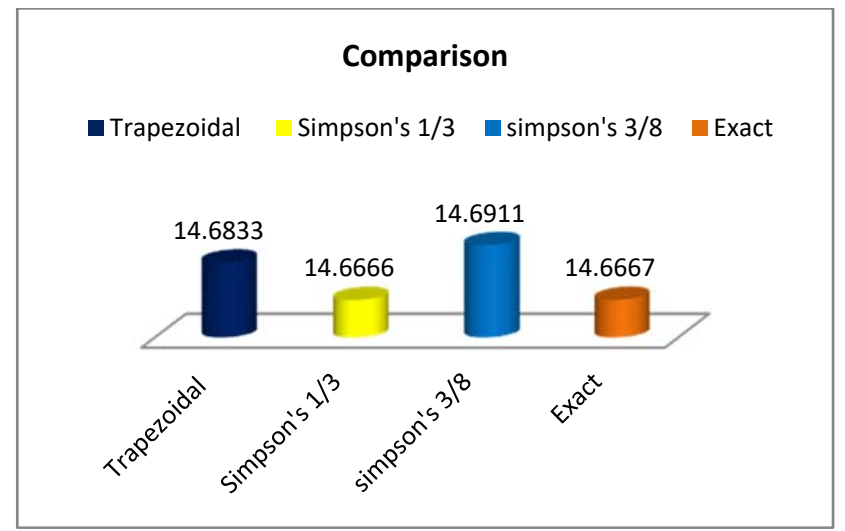

Fig.1. Comparison among Trapezoidal, Simpson's 1/3, Simpson's 3/8 and exact value

Table 3. Calculating the values of $f(x)$ for random values of $x$

\begin{tabular}{|c|c|c|c|c|c|}
\hline Value of $X$ & Value of $f(x)$ & Value of $X$ & Value of $f(x)$ & Value of $X$ & Value of $f(x)$ \\
\hline 0 & 0.111111111 & 0.31 & 0.114727928 & 0.64 & 0.127360295 \\
0.02 & 0.111125927 & 0.35 & 0.11574192 & 0.68 & 0.129619137 \\
0.03 & 0.111144449 & 0.38 & 0.116589842 & 0.69 & 0.130211648 \\
0.07 & 0.111292741 & 0.4 & 0.117197608 & 0.71 & 0.131430911 \\
0.09 & 0.111411516 & 0.42 & 0.11783994 & 0.73 & 0.132696699 \\
0.1 & 0.111482099 & 0.43 & 0.118174231 & 0.76 & 0.134685009 \\
0.14 & 0.111839413 & 0.45 & 0.118869408 & 0.78 & 0.136071968 \\
0.16 & 0.112063315 & 0.49 & 0.120368191 & 0.8 & 0.137509457 \\
0.19 & 0.112456222 & 0.5 & 0.120765926 & 0.84 & 0.140541068 \\
0.21 & 0.112756502 & 0.56 & 0.123352296 & 0.87 & 0.14295706 \\
0.23 & 0.113087736 & 0.57 & 0.123817525 & 0.91 & 0.146377161 \\
0.26 & 0.113643214 & 0.59 & 0.124778127 & 0.92 & 0.147268927 \\
0.29 & 0.114269954 & 0.61 & 0.125779599 & 0.96 & 0.150988861 \\
0.97 & 0.151958094 & 1.36 & 0.204887153 & 2.14 & 0.4684644461 \\
0.99 & 0.153945152 & 1.39 & 0.210456107 & 2.18 & 0.490539641 \\
1.04 & 0.159206769 & 1.48 & 0.228785423 & 2.26 & 0.537430254 \\
1.09 & 0.164912161 & 1.57 & 0.249801037 & 2.44 & 0.6543848 \\
1.16 & 0.173706705 & 1.64 & 0.268226838 & 2.79 & 0.888027287 \\
1.22 & 0.182060641 & 1.78 & 0.31129302 & $\pi$ & 0.9999997463 \\
1.27 & 0.189648749 & 1.93 & 0.367991082 & & \\
1.31 & 0.196159712 & 2.11 & 0.452525025 & & \\
& & & & \\
\end{tabular}

From Table 2 and Figure 1, we see that the value of Simpson's 1/3 formula is very close to exact value. i.e. Simpson's 1/3 formula provides more accurate result than Trapezoidal and Simpson's 3/8 formula for unequal space. 


\subsection{Investigation by Second Example}

Consider the integration

$$
f(x)=\int_{0}^{\pi} \frac{d x}{(2+\cos x)}
$$

We consider 60 sub intervals over the interval 0 to $\pi$

Table 3 represents the value of function $f(x)=\int_{0}^{\pi} \frac{d x}{(2+\cos x)}$ for different unequal values of $x$

Now, we determine the solution of the second problem using Trapezoidal, Simpson's $\frac{1}{3}$ and Simpson's $\frac{3}{8}$ rule for unequal data space and compare the result with exact result. The above task is given bellow:

Table 4. Comparison between exact and numerical result

\begin{tabular}{|l|l|c|l|}
\hline Method & Result & Exact value & Error \\
\hline Trapezoidal & 1.20146 & & $0.63927 \%$ \\
Simpsons $1 / 3$ & 1.20769 & 1.20919 & $0.12404 \%$ \\
Simpsons 3/8 & 1.22549 & & $1.3480 \%$ \\
\hline
\end{tabular}

Table 4 shows the comparison of Trapezoidal rule, Simpson's 1/3 and Simpson's 3/8 rule. Now, we describe the above comparison by the following diagram

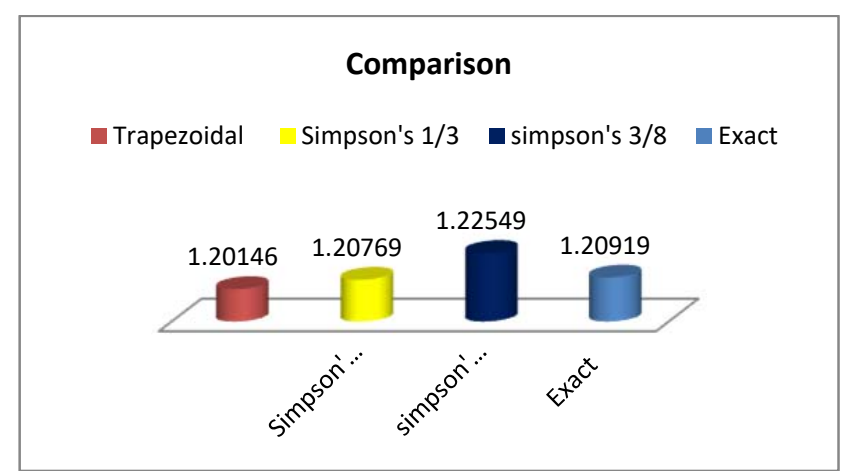

Fig.2. Comparison among Trapezoidal, Simpson's 1/3, Simpson's 3/8 and exact value

From Table 4 and Figure 2, we see that the value of Simpson's $1 / 3$ formula is very close to exact value. i.e. Simpson's $1 / 3$ formula provides more accurate result than Trapezoidal and Simpson's $3 / 8$ formula for unequal space.

\subsection{Investigation by Third Example}

Consider the integration 


$$
f(x)=\int_{0}^{1} x^{2} e^{x^{3}} d x
$$

We consider 60 subintervals over the interval 0 to 1

Table 5. Calculating the values of $f(x)$ for random values of $x$

\begin{tabular}{|c|c|c|c|c|c|}
\hline Value of $X$ & Value of $f(x)$ & Value of $X$ & Value of $f(x)$ & Value of $X$ & Value of $f(x)$ \\
\hline 0 & 0 & 0.27 & 0.074349105 & 0.53 & 0.325992943 \\
\hline 0.01 & 0.0001 & 0.28 & 0.080140066 & 0.56 & 0.373805007 \\
\hline 0.02 & 0.000400003 & 0.29 & 0.086176332 & 0.57 & 0.391001127 \\
\hline 0.04 & 0.001600102 & 0.3 & 0.092463102 & 0.59 & 0.427463453 \\
\hline 0.05 & 0.002500313 & 0.31 & 0.099005986 & 0.6 & 0.446796856 \\
\hline 0.06 & 0.003600778 & 0.32 & 0.105811024 & 0.62 & 0.487851803 \\
\hline 0.08 & 0.006403278 & 0.34 & 0.120234013 & 0.64 & 0.532362726 \\
\hline 0.09 & 0.008105907 & 0.35 & 0.127866408 & 0.66 & 0.580691939 \\
\hline 0.1 & 0.010010005 & 0.36 & 0.135789893 & 0.68 & 0.633248118 \\
\hline 0.11 & 0.012116116 & 0.38 & 0.152544938 & 0.7 & 0.690492693 \\
\hline 0.12 & 0.014424905 & 0.39 & 0.161395391 & 0.72 & 0.75294723 \\
\hline 0.14 & 0.019653856 & 0.4 & 0.170574784 & 0.73 & 0.786309743 \\
\hline 0.15 & 0.022576066 & 0.41 & 0.180094199 & 0.74 & 0.821201978 \\
\hline 0.17 & 0.029042335 & 0.42 & 0.189965437 & 0.76 & 0.895925792 \\
\hline 0.18 & 0.032589509 & 0.44 & 0.21081441 & 0.78 & 0.97787765 \\
\hline 0.19 & 0.036348461 & 0.45 & 0.221819699 & 0.8 & 1.06792007 \\
\hline 0.2 & 0.040321283 & 0.47 & 0.24506736 & 0.84 & 1.27634079 \\
\hline 0.21 & 0.044510307 & 0.49 & 0.270076298 & 0.9 & 1.679135317 \\
\hline 0.22 & 0.048918117 & 0.5 & 0.283287113 & 1 & 2.718281828 \\
\hline 0.23 & 0.053547566 & 0.51 & 0.296995555 & & \\
\hline 0.25 & 0.063484232 & 0.52 & 0.311223201 & & \\
\hline
\end{tabular}

Table 5 represents the value of $f(x)=\int_{0}^{1} x^{2} e^{x^{3}} d x$ for different unequal values of $x$

Now, we solve the third problem by using Trapezoidal, Simpson's $\frac{1}{3}$ and Simpson's $\frac{3}{8}$ rule for unequal data space and compare the result with exact result. The above procedure is given bellow:

Table 6. Comparison between exact and numerical result

\begin{tabular}{|c|c|c|c|}
\hline Method & Result & Exact value & Error \\
\hline Trapezoidal & 0.578427 & & $0.98941 \%$ \\
Simpsons 1/3 & 0.573508 & 0.57276 & $0.13059 \%$ \\
Simpsons 3/8 & 0.57901 & & $1.09120 \%$ \\
\hline
\end{tabular}

Table 6 represents the comparisons of Trapezoidal rule, Simpson's 1/3, Simpson's 3/8, rule and we see $\begin{array}{lllll}\text { that } & \text { Simpson's } & 1 / 3 & \text { gives } & \end{array}$ 
The above comparison is shown by the following diagram

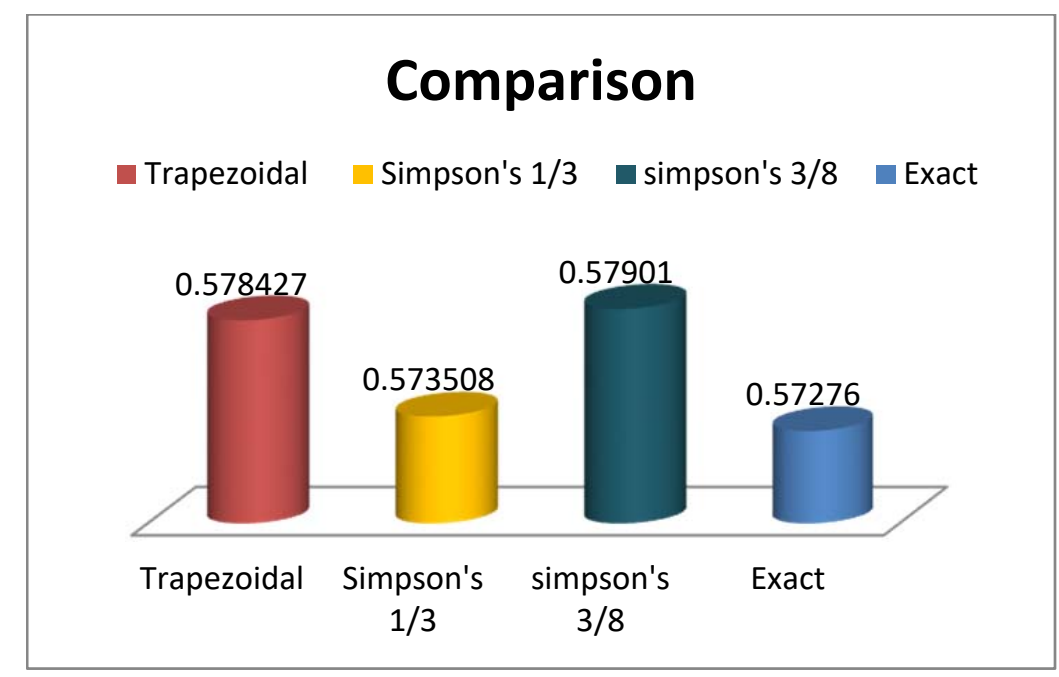

Fig.3. Comparison among Trapezoidal, Simpson's 1/3, Simpson's 3/8 and exact value

From Table 6 and Figure 3, we see that the value of Simpson's 1/3 formula is very close to exact value. i.e. Simpson's 1/3 formula provides more accurate result than Trapezoidal and Simpson's 3/8 formula for unequal space.

From above three examples we see that Simpson's 1/3 rule gives minimum error. So Simpson's $1 / 3$ rule is better than other methods for unequal space.

\section{Conclusion}

The main objective of our work is to determine better numerical integration formula for unequal data space. Therefore, we apply Trapezoidal rule, Simpson's 1/3 rule and Simpson's 3/8 rule to solve various numerical problems and compare the result with their exact solution. We have found that Simpson's $1 / 3$ rule gives better result than any other numerical method for unequal data space.

\section{References}

[1] Muthumalai R K. Some Formulae for Numerical Differentiation through Divided Difference. International Journal of Mathematical Archive. 2012, 03.

[2] Khan M M, Hossain M R and Selina parvin. Numerical Integration Schemes for Unequal Data Spacing. American Journal of Applied Mathematics. 2017, 5(2), 48-56.

[3] Thukral R. Further Acceleration of the Simpson's Method for Solving Non-linear Equation. Journal of Advances in Mathematics. 2018, 14, 02.

[4] Jayakumar J. Generalized Simpson-Newton's Method for Solving Nonlinear Equations with Cubic Convergence. IOSR Journal of Mathematics. 2013, 7(5), 58-61.

[5] Atkinson K E. An Introduction to Numerical Analysis, John Wiley \& Sons, New York. 1978.

[6] Conte S D. Elementary Numerical Analysis. McGraw Hill, New York. 1965.

[7] Curtis F G, Patrick O. Wheatley. Applied Numerical Analysis. Pearson Education, Inc. 2004. 
[8] Gill P E, Miller G F. An Algorithm For the Integration of Unequally Spaced Data. The Computer Journal. 1972, 15, 80-83.

[9] Cheney W, David Kincaid. Numerical Mathematics and Computing. Thomson Brooks/Cole. 2008.

[10] Faires J D, Burden L R. Numerical Analysis, Thomson Learning. 2001.

[11] Hildebrand F B, Graw-Hill M C. Introduction to Numerical Analysis. 2nd Edition, New York. 1974.

[12] Eskandari H. Simpson's Method Solution for Non-linear equation, Scientific Research Publishing Inc. 2017, 8, 929-933.

[13] Liang X P. A Numerical Integration Method of Dynamic Finite Element Analysis. Scientific Net. Advanced Materials Research, 2011, 199-200, 1257-1261.

[14] Hou J, Kui Liu and Qian Mu Li. Analysis on Calculating Numerical Integration Methods of Common Social Science Field. Scientific Net, Applied Mechanics and Materials. 2012, 121-126, 4391-4395.

[15] Filiz A. Numerical Solution of a Non-linear Volterra Integro differential Equation via Runge-KuttaVerner Method. International Journal of Scientific and Research Publications. 2013, 3 (9), 1-8.

[16] Kambo N S. Error of the Newton-Cotes and Gauss-Legendre Quadrature Formulas, Mathematics of Computation. 1970, 24, 110.

[17] Sinha R K and Kumar R. Numerical method for evaluating the integrable function on a finite interval. International Journal of Engineering Science and Technology. 2010, 2(6).

[18] Sozio G. Numerical Integration. Australian Senior Mathematics Journal. 2009, 23(1).

[19] Mantzaris N V, Daoutidis P and Srienc F. Numerical solution of multi-variable cell population balance models: I. Finite difference methods. Computers \& Chemical Engineering. 2001, 25(11-12), 1411-1440.

[20] Abia L M, Angulo O, Lopez-Marcos J C and Lopez-Marcos M A. Numerical integration of a hierarchically size- ' structured population model with contest competition. Journal of Computational and Applied Mathematics. 2014, 258, 116- 134. 


\section{Authors' Profiles}

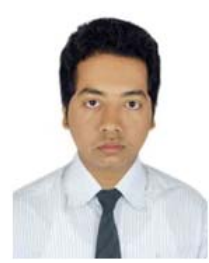

Md. Nayan Dhali completed B S (Honor's) and M S in Mathematics from the University of Dhaka. He is currently working as a lecturer in the Department of Mathemtics, Jashore University of Science and Technology, Jashore, Bangladesh. His research interest includes numerical analysis.

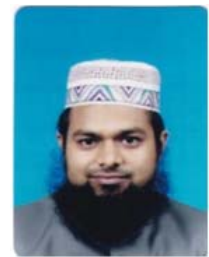

Mohammad Farhad Bulbul is currently working as an Assistant Professor in the Department of Mathematics at Jashore University of Science and Technology, Jashore. He received his $\mathrm{PhD}$ degree from the Department of Information Science, Peking University, Beijing, China, in 2016. His $\mathrm{PhD}$ research direction was "Statistical Learning and Intelligent Information Processing". He achieved the Peking University President's award for his outstanding performance in doctoral study (Including course work and research). From 2012 to 2016, he held the research assistantship position with the school of Mathematical Sciences, Peking University. He has published some papers in SCIE indexed journals (Clarivate Analytics journals), IEEE International Conference Proceedings (EI Indexed) and Lecture Notes in Computer Science (Springer-Verlag, EI Indexed). His research focuses on Computer Vision, Deep Learning, Pattern Recognition, and Image Processing.

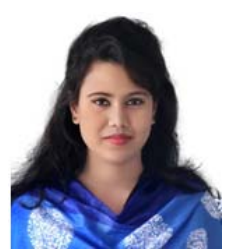

Umme Sadiya completed her B.Sc. (Honor's) in Mathematics from the Department of Mathematics, Jashore University of Science and Technology, Jessore, Bangladesh. She is currently pursuing M.S. in Applied Mathematics at the department. Her research interest includes numerical analysis.

How to cite this paper: Md. Nayan Dhali, Mohammad Farhad Bulbul, Umme Sadiya," Comparison on Trapezoidal and Simpson's Rule for Unequal Data Space", International Journal of Mathematical Sciences and Computing(IJMSC), Vol.5, No.4, pp.33-43, 2019. DOI: 10.5815/ijmsc.2019.04.04 